\title{
ESTRATEGIAS PARA EL MEJORAMIENTO DE LA CALIDAD AMBIENTAL EN LA COMUNA TRES, SAN JUAN DE PASTO
}

\author{
STRATEGIES FOR THE IMPROVEMENT \\ OF ENVIRONMENTAL QUALITY IN COMMUNE THREE, \\ SAN JUAN DE PASTO
}

\section{${ }^{1}$ David Andrés Calvache M, ${ }^{2}$ Juan Camilo Pejendino S, ${ }^{3}$ Álvaro Javier Ceballos F}

${ }_{1}^{1}$ Magister en Agroforestería Tropical. Universidad de Nariño, Docente Tiempo Completo. Universidad de Nariño. Pasto, Colombia.

${ }^{2}$ Ingeniero Ambiental. Universidad de Nariño. Pasto, Colombia.

3Magister en Desarrollo regional y planificación del territorio. Docente hora cátedra. Universidad de Nariño. Pasto, Colombia.

${ }^{1}$ dacalvache@udenar.edu.co, ${ }^{2}$ juanp@udenar.edu.co, ${ }^{3}$ aceb1980@udenar.edu.co

\section{RESUMEN}

Contextualización: la ciudad de San Juan de Pasto, en especial su área céntrica y suroriental, presenta una presión significativa en los recursos ambientales a causa de una mayor densidad poblacional, lo que genera diversos problemas ambientales a razón del proceso de urbanización.

Vacío de investigación: es necesario formular estrategias que contribuyan al mejoramiento de la calidad ambiental urbana en la comuna tres, por medio de la evaluación de indicadores de calidad ambiental urbana, la estimación de la calidad ambiental en la zona de estudio y la formulación de alternativas que aumenten dicho componente mitigando impactos ambientales.

Propósito del estudio: los objetivos específicos del estudio fueron: identificar indicadores de calidad ambiental, estimar el índice de calidad ambiental urbana y proponer alternativas para mejorar la calidad ambiental urbana en la comuna tres, San Juan de Pasto.

Metodología: se aplicó el índice de calidad ambiental urbana, metodología diseñada por el Ministerio de Ambiente y Desarrollo Sostenible, como un instrumento para evaluar la sostenibilidad ambiental en las urbes. Se realizó 
la priorización de alternativas a través del método matriz de priorización multicriterio.

Resultados y conclusiones: el valor del índice de calidad ambiental urbana para la comuna tres obtuvo un valor de 50,5, lo cual advierte de una calidad ambiental urbana media. Los indicadores más representativos son: calidad del aire y porcentaje de población urbana localizada en zonas de amenaza alta, obteniendo una calificación de muy alto y muy bajo, respectivamente. La priorización de alternativas dio lugar al estudio de dos estrategias, un sistema de terrazas verdes modulares y una estrategia de movilidad sostenible - Sistema público de bicicletas, que mejorarían la calidad ambiental del área de estudio. Este proceso concluyó que la aplicación del índice de calidad ambiental urbana tiene una viabilidad moderada en la aplicación al nivel estratégico de comuna.

Palabras Clave: análisis multicriterio; área urbana; indicadores de calidad Ambiental; movilidad sostenible; techo verde.

\section{ABSTRACT}

Contextualization: This study identifies that the city of San Juan de Pasto, especially its central and southeastern area, present a significant pressure on environmental resources, due to a higher population density, generating various environmental problems due to the urbanization process.

Knowledge gap: Such situation gave rise to the development of this research, to formulate strategies that contribute to the improvement of urban environmental quality in commune three, by obtaining urban environmental quality indicators, estimating environmental quality in the area of study and formulation of alternatives that increase urban environmental quality and prevent environmental impacts.

Purpose: The specific objectives of the study were: Identify environmental quality indicators, estimate the urban environmental quality index and propose alternatives to improve urban environmental quality in commune three, San Juan de Pasto. For this, the urban environmental quality index, a methodology designed by the Ministry of Environment and Sustainable Development (2016), was applied as an instrument to assess environmental sustainability in cities and prioritization of alternatives was carried out through of the method; multi-criteria prioritization matrix.

Results and conclusions: The urban environmental quality index value for commune 3 obtained a rating of 50.5 , which indicates an average urban environmental quality, with Air Quality and the percentage of urban population located in high threat areas being among the most representative, obtaining a Very high and very low rating respectively. The prioritization of alternatives led to the study of two strategies; a modular green terrace system and a sustainable mobility strategy Public bicycle system, which would improve the environmental quality of the study area. This process concluded that the application of the urban environmental quality index proposed in previous research has a moderate viability in the application at the strategic level of the commune.

Key words: Multi-criteria analysis; urban area; Environmental quality indicators; sustainable mobility; green roofs. 


\section{RESUMEN GRÁFICO}

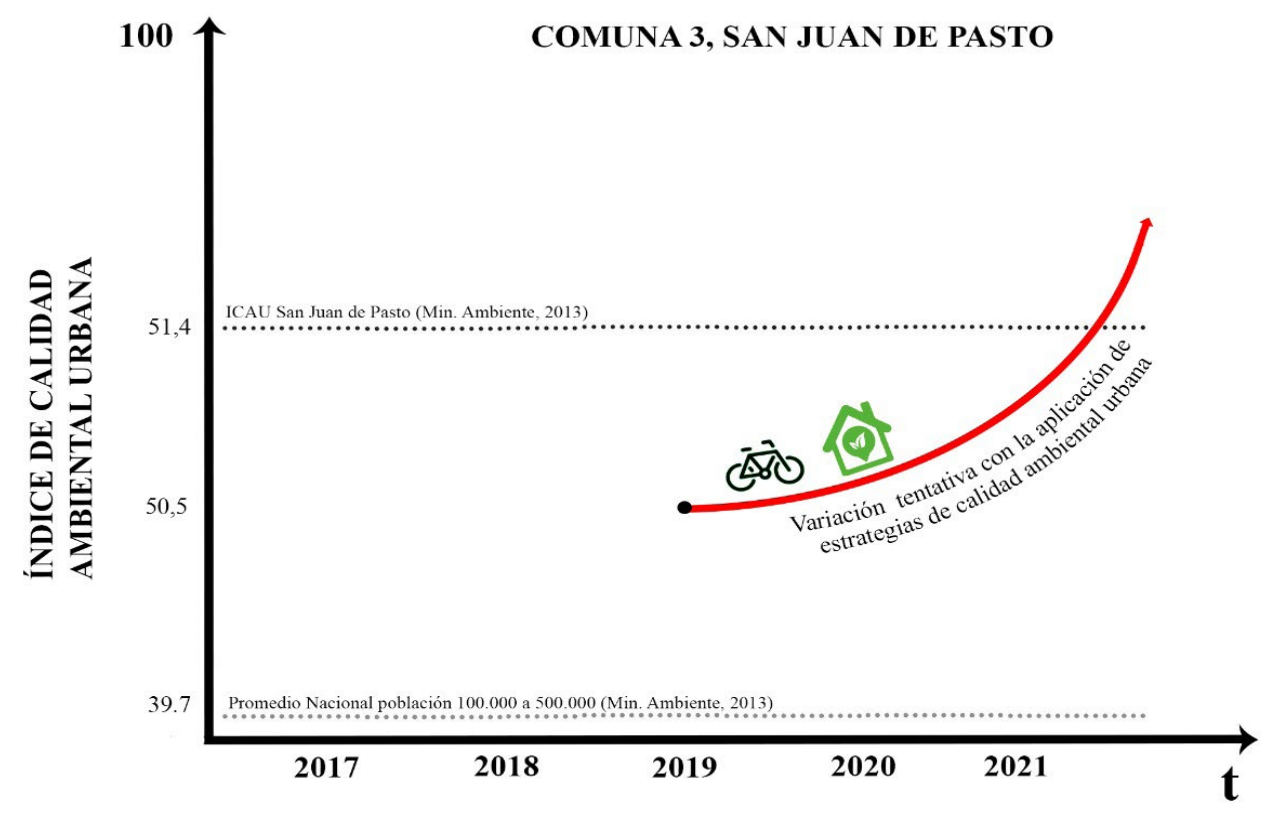

Fuente: Autores

\section{INTRODUCCIÓN}

Las ciudades o áreas urbanas se definen como la conformación de conjuntos de edificaciones y estructuras contiguas agrupadas en manzanas que están delimitadas por calles, carreras o avenidas, y que prestan servicios básicos tales como acueducto, alcantarillado, energía eléctrica, hospitales y colegios (Charry y Gallego, 2018). En dichas áreas también concurren interacciones sociales, económicas y ambientales, dentro de las que se contempla la calidad ambiental urbana, entendida ésta última como la interacción de un conjunto de factores humanos y ambientales interrelacionados que inciden favorable o desfavorablemente en la calidad de vida de los habitantes de una ciudad (Rojas, 2011).

Las dinámicas de crecimiento demográfico que enfrentan las ciudades representan una alta amenaza para el medio ambiente, para la salud y la calidad de vida de sus habitantes. Dicho crecimiento genera nuevos procesos económicos y está generalmente acompañado de un incremento en las actividades industriales, mayores tasas de motorización, superior consumo de combustible $y$, por ende, la generación de mayores emisiones de contaminantes del aire (Franco, 2012).

Actualmente, el $54 \%$ de la población mundial vive en zonas urbanas y se espera que en 2050 alcance el 66\% (Bocquier, 2005). En el caso de Colombia, la población urbana, en el año 2014, representa el 76\% y, según las proyecciones, se estima que para 2050 la población llegará a 52,6 millones de habitantes, lo que equivale al $86 \%$ de la población total (MundialDNP, 2014). En Colombia, durante el año 2014, el $75 \%$ de la población se ha concentrado en suelos urbanos que corresponden tan solo al $1,2 \%$ del área total del territorio nacional. Entre estos, se destacan grandes aglomeraciones de población en ciudades como Bogotá D.C., Medellín, Cali y Barranquilla, las cuales sobresalen respecto a otros centros poblados 
por sus actividades socioeconómicas (IGAC, 2015; Instituto de Estudios Urbanos, 2017).

Según la Alcaldía de Pasto (2014), en el POT 2014-2027, el municipio de Pasto cuenta con una extensión de $109.555 \mathrm{Ha}$, conformado por 107.188 Ha de área rural, siendo el 97,8\% del área total del municipio. En el año 1938, la distribución de la población era casi equitativa en los sectores urbano y rural; sin embargo, se observa una tendencia hacia la disminución de la población rural y el aumento de la población urbana. Para el 2020, la población urbana alcanza el $84 \%$ de la población total del municipio. Según la Alcaldía de Pasto (2014), en el año 2012 la comuna tres presentó una densidad adecuada, pese a poseer terrenos destinados a viviendas unifamiliares de interés social. En el año 2014, la densidad poblacional del área urbana de San Juan de Pasto fue de 197 hab/ha; no obstante, la comuna tres cuenta con 43.828 habitantes y una densidad igual a 431 hab/ha, posicionándose como la comuna con mayor densidad poblacional y número de habitantes, esto debido a un dinamismo acelerado y una consolidación importante dentro del territorio en los últimos años (Alcaldía de Pasto, 2014).

La importancia de esta investigación radicó en formular estrategias que contribuyan al mejoramiento de la calidad ambiental urbana y disminuyan impactos ambientales en la comuna 3 de la ciudad de San Juan de Pasto causados, en parte, por la ausencia de la planificación en el proceso urbanizador que ocasionó el crecimiento desorganizado en la ciudad de San Juan de Pasto (Figueroa y Ordoñez, 2014).

Este estudio logró una estimación objetiva del estado actual de la calidad ambiental, en la comuna tres de la ciudad San Juan de Pasto, a través de la evaluación de indicadores ambientales directos e indirectos. Los objetivos específicos por desarrollar en este estudio fueron: obtener la calificación de los indicadores de calidad ambiental urbana propuestos por el Ministerio de Ambiente y Desarrollo Sostenible (2016), para la comuna tres de la ciudad de Pasto, estimar la calidad ambiental en la zona de estudio y formular alternativas que mejoren la calidad ambiental urbana y mitiguen impactos ambientales, en base en la estimación del ICAU.

Este estudio establece la formulación y priorización de dos estrategias: un sistema de terrazas verdes modulares y una estrategia de movilidad sostenible (sistema público de bicicletas), sin embargo, deja abierta la oportunidad de la posible ejecución de las estrategias, mejorando la valoración de indicadores ambientales en la comuna tres, así como de la región en general, aportando en la disminución de la cantidad de enfermedades de tipo respiratorio, mejorando la belleza paisajística y previniendo la variabilidad climática.

\section{MATERIALES Y MÉTODOS Localización del área de estudio}

El estudio se realizó en la ciudad de San Juan de Pasto, específicamente en la comuna tres, ubicada al sur oriente, siguiendo la vía de acceso al departamento del Putumayo, entre las comunas dos, doce y cuatro; colindando con los corregimientos de Buesaquillo y Mocondino. Esta comuna está ubicada en las coordenadas $1^{\circ} 12^{\prime} 13^{\prime \prime} \mathrm{N}$ y $77^{\circ} 15^{\prime} 24^{\prime \prime} \mathrm{W}$. Según la Oficina de Cabildos Indígenas - Alcaldía de Pasto (2013) en Pinto (2013). En ella habitan 56.761 individuos. En la figura 1, se indica la localización geográfica de la comuna tres en la ciudad de San Juan de Pasto. 
MAPA DE LOCALIZACIÓN ÁREA DE ESTUDIO
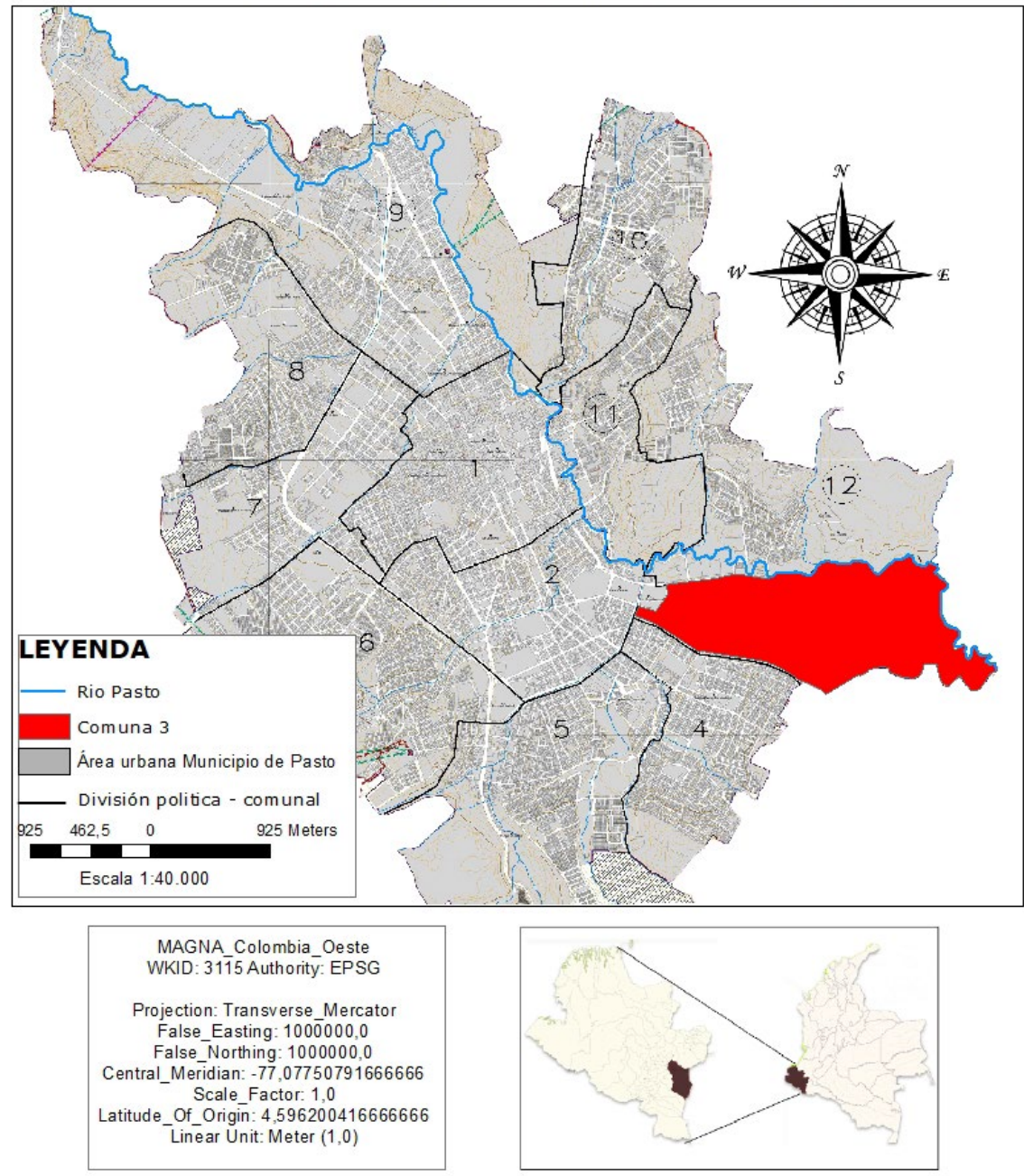

Figura 1. Localización geográfica de la comuna 3 de la ciudad de San Juan de Pasto.

Este estudio. Apoyado con Datos POT 2014 - 2027.

Fuente: Alcaldía de Pasto (2014)

Indicadores de calidad ambiental urbana Para lograr la calificación de indicadores de la calidad ambiental urbana, se usó la metodológica ICAU de urbes propuesta por el Ministerio de Ambiente y Desarrollo Sostenible, en el año 2016, con nivel de complejidad urbana entre 100.000 y 499.999 habitantes. Aunque el área de estudio está compuesta por 56.761 habitantes, se selecciona ese nivel de complejidad debido a que la comuna tres está vinculada en directas relaciones económicas, sociales y culturales con las doce comunas de San Juan de Pasto. En la Tabla 1 se indican las fórmulas para el cálculo de los indicadores. 
Tabla 1. Cálculo de indicadores.

\begin{tabular}{|c|c|c|}
\hline $\mathbf{N}^{\circ}$ & Fórmula. & Descripción. \\
\hline 1 & $A V U p c=\frac{A V U}{P U T}$ & $\begin{array}{l}\text { AVUpc: área verde por habitante }\left(\mathrm{m}^{2}\right) \\
\text { AVU: total de áreas verdes urbanas en comuna }\left(\mathrm{m}^{2}\right) \\
\text { PUT: población urbana total (por comuna) (número de personas) }\end{array}$ \\
\hline 2 & $\% E C=\frac{E c}{E t} * 100$ & $\begin{array}{l}\text { \%EC: porcentaje de estaciones que reportan concentraciones anuales de } \mathrm{PM}_{10} \\
\text { y } \mathrm{PM}_{2,5} \text {, normativa vigente. } \\
\text { Ec: número de estaciones que reportan concentraciones anuales de } \mathrm{PM}_{10} \text {, } \\
\text { normativa vigente. } \\
\text { Et: total de estaciones monitoreando } \mathrm{PM}_{10} \text { y } \mathrm{PM}_{2,5} \text { en el periodo de tiempo (t) } \\
\text { del área de cada una de las comunas. }\end{array}$ \\
\hline 3 & $I C A_{i, j}=\sum_{i=1} W_{i} I_{i j t}$ & $\begin{array}{l}\text { ICA }_{j, t} \text { : índice de calidad el agua para una determinada corriente superficial en } \\
\text { la estación de monitoreo } j \text {, en el tiempo } t \text {. } \\
\mathrm{W}_{\mathrm{i}} \text { : peso o ponderador asignado a cada variable de calidad } \mathrm{i} \text {. } \\
\mathrm{I}_{\mathrm{ijt}} \text { : subíndice de calidad de la variable } \mathrm{i} \text {, de acuerdo con las curvas funcionales } \\
\text { o ecuaciones correspondientes, en la estación de monitoreo } \mathrm{j} \text {, en el tiempo t. }\end{array}$ \\
\hline 4 & $\% R S A=\left(\frac{\Sigma R S A}{R S G}\right) * 100$ & $\begin{array}{l}\text { RSG (Ton) = RS dispuestos (Ton) + RS aprovechados (Ton) } \\
\text { RSA: RS Aprovechados (Ton) }\end{array}$ \\
\hline 5 & $P U A R=A U E P * F D P$ & $\begin{array}{l}\text { PUAR: población urbana expuesta a ruido ambiental por encima del valor de } \\
\text { referencia }(65 \mathrm{~dB}) \\
\text { AUEP: área de las zonas en las cuales se determinó incumplimiento del valor } \\
\text { de referencia del nivel equivalente día noche de } 65 \mathrm{~dB}(\mathrm{~A}) \text {, en kilómetros } \\
\text { cuadrados. ( } \mathrm{Km} 2) \\
\text { FDP: factor de densidad poblacional. }\end{array}$ \\
\hline 6 & $\% D N M=\frac{C R A P H}{\text { Dotación RAS }}$ & $\begin{array}{l}\text { \% DNM: Porcentaje de consumo de agua con relación a la dotación neta } \\
\text { máxima establecida en el RAS } \\
\text { CRAPH: consumo residencial de agua por habitante [l/hab-día] } \\
\text { Dotación RAS: dotación neta máxima establecida en el RAS según nivel de } \\
\text { complejidad y clima [I/hab-día] }\end{array}$ \\
\hline 7 & $\% C E=\frac{C R E P H 1}{C R E P H 2} * 100$ & $\begin{array}{l}\text { \%CE: porcentaje de consumo residencial. } \\
\text { CREPH1: consumo residencial de energía por habitante (Kwh/ habitante/ año) } \\
\text { (Año de reporte ICAU) } \\
\text { CREPH2: consumo residencial de energía por habitante (Kwh/ habitante/ año) } \\
\text { (Año reporte ICAU anterior) }\end{array}$ \\
\hline 8 & $\% R S D=\frac{R S D_{j . t}}{R G_{j . t}} * 100$ & $\begin{array}{l}\text { \% RSD: porcentaje de residuos sólidos generados en el área urbana y que } \\
\text { son dispuestos adecuadamente } \\
\text { RSDjt: cantidad de RS dispuestos adecuadamente (Ton) } \\
\text { RGjt: cantidad de RS generados (RS dispuestos + RS aprovechados) (Ton) }\end{array}$ \\
\hline 9 & $C U S P U=\frac{S P U C U}{S P U T} * 100$ & $\begin{array}{l}\text { CUSPU: porcentaje del suelo de protección urbano con conflictos de uso del } \\
\text { suelo (\%). } \\
\text { SPUCU: superficie del suelo de protección urbano con conflictos de uso del } \\
\text { suelo (Ha). } \\
\text { SPUT: superficie del Suelo de protección urbano }(\mathrm{Ha}) \text {. }\end{array}$ \\
\hline
\end{tabular}

Fuente: Ministerio de Ambiente y Desarrollo Sostenible (2016), 
A continuación, se describe la metodología de cada indicador de calidad ambiental urbana:

Superficie de área verde por habitante: para obtener la cuantificación de la superficie de área verde por habitante, se tomó como base el plano N.O EE2 - Tratamientos Urbanísticos - del plan de ordenamiento territorial 2014 -2027 (Alcaldía de Pasto, 2014) donde se indican las zonas verdes. Se procedió a actualizar la información mediante la rasterización y digitalización de imágenes Landsat, y de imágenes capturadas con dron en sobrevuelo del área de estudio, tal como se muestra en la figura anexo $\mathrm{N}^{\circ} 1$. Con el valor de la extensión de superficie de área verde se procedió a realizar el cálculo del área verde por habitante a través de la ecuación N. ${ }^{\circ} 1$ de la tabla 1 , teniendo en cuenta la densidad poblacional como base para el cálculo.

Calidad del aire: se usó la información brindada por CORPONARIÑO (S.f.) y la plataforma gubernamental IDEAM (2018) de la estación de monitoreo de calidad de aire ubicada en la Universidad Mariana. El cálculo del indicador de calidad de aire se realizó con base en la evaluación de PM10 de los años 2017 y 2018. Se realizó el cálculo de calidad de aire con base en la ecuación $N^{\circ} 2$ de la tabla 1.

Calidad del agua superficial: para obtener este indicador se usó la información contenida en el Plan de Ordenamiento del Cauce Principal del Río Pasto (CORPONARIÑO, 2011). Específicamente las caracterizaciones fisicoquímicas de los tramos antes de la Quebrada Dolores y el tramo Bocatoma Centenario, como se indica en la figura Anexo $\mathrm{N}^{\circ} 2$. La calidad de agua superficial se basó de los parámetros: sólidos suspendidos totales, $\mathrm{pH}$, oxígeno disuelto, demanda química de oxígeno, nitrógeno total y fósforo total. Se obtuvo la calidad de agua superficial a través de la ecuación $N^{\circ} 3$ de la tabla 1.
Porcentaje de áreas protegidas: se le solicitó información a la Alcaldía Municipal de Pasto y el análisis de áreas protegidas declaradas para el municipio de San Juan de Pasto mediante las resoluciones: Res. 205 de agosto 22/2003, Res. 129 de noviembre 12/2014, Res. 163 de diciembre 6/2006, Acuerdo 5 de febrero 24/1971, Res. 009 de mayo 19/1943 y Res. 052 de marzo 22/1985 según el PGAR 2016 - 2036 (CORPONARIÑO, 2016). Se comparó si las áreas protegidas se encuentran en solapamiento con la comuna 3.

\section{Porcentaje de residuos sólidos aprove-} chados: se revisó y recopiló información del Plan de Gestión Integral de Residuos Sólidos 2015 - 2027 de la Alcaldía Municipal de Pasto. El cálculo del indicador se realizó con base a proyecciones del PGIRS para el año 2019 en valores de residuos sólidos y residuos sólidos aprovechados. Dicho cálculo se obtuvo gracias a la aplicación de la ecuación No 4 de la tabla 1.

\section{Porcentaje de población urbana expuesta} a ruido por encima del nivel de referencia: para el cálculo de este indicador, se tomó como base el mapa de ruido ambiental para el área urbana del Municipio de Pasto, contenido en el informe de gestión de la vigencia 2014 (CORPONARIÑO, 2015) el cual se digitalizó y se intersecó con la comuna 3 como se indica en la Figura Anexo N.o 3. Posteriormente se calculó el área que presenta niveles de ruido superior a $65 \mathrm{~dB}$ y teniendo en cuenta la densidad poblacional se encontró el número de personas que se ve afectada por ruido por encima del nivel de referencia. Este cálculo se realizó con base en la ecuación No 5 de la tabla 1.

Porcentaje de población vinculada a estrategias de educación ambiental en áreas urbanas y Porcentaje de población que participa en gestión ambiental urba-

na: para el cálculo de estos indicadores, fue 
necesario encuestar a 268 personas de la comuna 3, que representan una muestra significativa con un margen de error del 5,79\% y un nivel de confianza equivalente al 94,31\%, calculados con base en la ecuación [10] Surveymonkey (S.f.):

$$
\text { Tamaño de muestra }=\frac{\frac{z^{2} * p(1-p)}{e^{2}}}{1+\left(\frac{z^{2} * p(1-p)}{e^{2} N}\right)}[\text { Ecuación 10] }
$$

Donde:

Z: Puntuación del nivel de confianza deseado

$\mathrm{N}$ : Tamaño de la población

e: Margen de error

P: población característica del estudio

Posterior a esto, se organizaron los resultados en una tabla de Excel y se hizo el cálculo de los porcentajes.

\section{Porcentaje de población urbana localiza-} da en zonas de amenaza alta: para el cálculo de este indicador, fue necesario el análisis del mapa de amenazas por fenómenos de origen natural y socio natural generado por el Servicio Geológico Colombiano (2015). Se realizó un traslape de la comuna tres con el área de amenaza volcánica alta, para evidenciar la existencia de áreas de amenaza alta en la comuna 3, tal como se indica en la Figura Anexo N.o 4.

\section{Consumo residencial de agua y energía} por habitante: para el cálculo de estos indicadores fue necesario revisar y extraer información del Informe Nacional de Calidad Ambiental Urbana (Ministerio de Ambiente y Desarrollo Sostenible, 2016) y posteriormente se realizó la actualización de valores con base en el Informe de Gestión 2019 generado por la empresa CEDENAR S.A. E.S.P (2019). Para el cálculo de estos indicadores fue necesario aplicar las fórmulas No 6 y 7 de la tabla 1 .
Porcentaje de residuos sólidos dispuestos adecuadamente: EMAS S.A. E.S.P (2018) dio lugar al cálculo del porcentaje de residuos sólidos dispuestos adecuadamente a través del Informe de Gestión de EMAS Pasto 2019. Calculado a partir de la Ecuación No 8 de la tabla 1.

\section{Porcentaje de suelos de protección urba-} nos: se realizó la digitalización de los suelos de protección urbanos con base en el plano EE7 - Planes Parciales, contenido en el POT del municipio de Pasto (Alcaldía Municipal de Pasto, 2014), que contenía la información de las áreas urbanas con clasificación de suelos de protección. Esta información se intersecó con la comuna 3, dando lugar a la observación del área que presenta suelos de protección urbana y logrando cuantificar el porcentaje de suelos de protección urbanos, tal como se muestra en la Figura Anexo No 5. Dicho porcentaje se calculó con base en la ecuación No 9 de la tabla 1.

Espacio público efectivo por habitante: el cálculo de este indicador se logra a partir de revisión bibliográfica en la biblioteca Alberto Quijano Guerrero de la Universidad de Nariño, del trabajo de grado (tesis de pregrado) titulada: Identificación de áreas óptimas para cubrir el déficit de espacio público en las comunas 3,4 y 5 de la ciudad de San Juan de Pasto (Montenegro y Delgado, 2015). 
Estimación del índice de calidad ambiental urbana (ICAU): para estimar el Índice de Calidad Ambiental Urbana, el Ministerio de Ambiente y Desarrollo Sostenible, 2016 establece que el grupo de indicadores directos debe tener un mayor peso en la definición del resultado final del ICAU, por lo cual cuenta con una participación del $70 \%$ y el $30 \%$, lo representa el grupo de indicadores indirectos. Al interior de cada grupo de indicadores, directos e indirectos, se debe otorgar el mismo porcentaje de participación a cada uno de los indicadores que lo conforman, por lo cual el valor del grupo se estableció utilizando las formulas 11 y 12 establecidas en la tabla 2.

Tabla 2. Estimación del índice de calidad ambiental urbana.

\begin{tabular}{|c|c|c|}
\hline Ecuaciones 11 y 12 & Descripción & Fuente. \\
\hline$v F D=\sum V O I * \frac{70}{\# \text { de indicadores del grupo }}[$ Ecuación 11] & $\begin{array}{l}\text { Valor final del grupo } \\
\text { de indicadores } \\
\text { directos. }\end{array}$ & \multirow{3}{*}{$\begin{array}{l}\text { Ministerio } \\
\text { de ambiente } \\
\text { y desarrollo } \\
\text { sostenible } \\
\text { (2016) }\end{array}$} \\
\hline$v F I=\sum V O I * \frac{30}{\# \text { de indicadores del grupo }}[$ Ecuación 12] & $\begin{array}{l}\text { Valor final del grupo } \\
\text { de indicadores } \\
\text { indirectos }\end{array}$ & \\
\hline$I C A U=V F D+V F I$ & $\begin{array}{l}\text { Índice de Calidad } \\
\text { Ambiental Urbana. }\end{array}$ & \\
\hline
\end{tabular}

Fuente: adaptada de: Índice de calidad ambiental urbana 2016 (Ministerio de Ambiente y Desarrollo Sostenible, 2016)

Una vez obtenidos los valores finales (VF) de cada grupo de indicadores (directos e indirectos), se realiza la suma de los dos valores, cuyo resultado es el valor final del Índice de calidad ambiental (Ministerio de Ambiente $y$ Desarrollo Sostenible, 2016).
Para su interpretación, el resultado obtenido se debe clasificar en uno de los cinco (5) rangos definidos en la tabla 3, donde cada uno de ellos indica un nivel de calidad ambiental urbana.

Tabla 3. Ponderación de valores y definiciones para resultado final de ICAU.

\begin{tabular}{l|l} 
Calidad Ambiental Urbana & \multicolumn{1}{c}{ Puntaje } \\
\hline Muy Baja & $<$ a 20 puntos \\
\hline Baja & 20,1 a 40 puntos \\
\hline Media & $\mathbf{4 0 , 1}$ a 60 puntos \\
\hline Alta & 60,1 a 80 puntos \\
\hline Muy Alta & $>$ a 80 puntos \\
\hline
\end{tabular}

Fuente: índice de calidad ambiental urbana, Ministerio de Ambiente y Desarrollo Sostenible 2016 (Ministerio de Ambiente y Desarrollo Sostenible, 2016). 


\section{Matriz de priorización multicriterio}

La formulación de las estrategias para mejorar la calidad de los indicadores ambientales se realizó a través de una matriz de priorización multicriterio, por el método de suma ponderada propuesta por Aznar y Guijarro (2012). La suma ponderada permite ponderar una serie de alternativas en función de un grupo de criterios, donde se tiene el valor normalizado, $\mathrm{X}_{\mathrm{ij},}$ de las variables para cada alternativa y el peso o ponderación de cada variable, $\mathrm{W}_{\mathrm{j}}$. Tal como se muestra en la ecuación [13].

$$
W_{i}=\sum_{j=1}^{n}\left(w_{j} * x_{i j}\right) \text { [Ecuación 13] }
$$

Siendo:

$\mathrm{W}_{\mathrm{i}}$ : ponderación final obtenida de cada alternativa

$\mathrm{W}_{\mathrm{j}}$ : peso de cada variable obtenido por la ponderación

$\mathrm{X}_{\mathrm{ij}}$ : valor de cada variable para cada alternativa.

Para esta investigación se establecieron los siguientes criterios: impacto ambiental, alcance, viabilidad, criterio financiero y prioridad. Estos criterios se seleccionaron a partir del estudio, análisis y retroalimentación entre los investigadores y habitantes de la comuna tres. Dichos criterios se valoran para cada alternativa de cero a diez y se realiza la obtención del peso de cada variable a través del método CRITIC de Diakoulaki, Mavrotas y Papayannakis (1995). El valor de la calificación para cada alternativa estará relacionado con el nivel de priorización.

\section{Planteamiento de estrategias}

La formulación de estrategias ambientales se llevó a cabo a través del análisis, planteamiento y discusión de medidas de protección ambiental que tengan como propósito contribuir al mejoramiento de la calidad ambiental. El modelo de proceso estratégico enfatizó el carácter emergente e innovador de la estrategia, así como su carácter eminentemente organizativo. Se plantearon estrategias que mejoraran la calidad ambiental, las cuales tuvieran referencias nacionales e internacionales y que logren discutir asertivamente los resultados de dichas estrategias.

\section{RESULTADOS Y DISCUSIÓN}

\section{Calificación de los indicadores de calidad ambiental urbana}

El análisis de la cartografía, la generación de los valores de área de cobertura vegetal, la recopilación de información en campo y demás aplicaciones de las ciencias ambientales dan lugar al cálculo de los indicadores de calidad ambiental urbana, mediante la metodología descrita por el Ministerio de Ambiente y Desarrollo Sostenible, 2016. Se logró compilar la información en la tabla 4, tal como se indica a continuación: 
Tabla 4. Resultados ICAU - Comuna 3 del Municipio de Pasto.

\begin{tabular}{|c|c|c|c|c|}
\hline \multicolumn{2}{|c|}{ Indicador } & \multirow{2}{*}{$\begin{array}{c}\text { Calificación con } \\
\text { base en el ICAU } \\
0,5\end{array}$} & \multirow{2}{*}{$\begin{array}{c}\text { Clasificación } \\
\text { Medio }\end{array}$} & \multirow{2}{*}{$\begin{array}{l}\text { Fuente. } \\
\text { Este estudio, 2019. Apoyado con } \\
\text { Datos POT, Alcaldía de Pasto (2014) }\end{array}$} \\
\hline \multirow{9}{*}{$\begin{array}{l}\mathbf{D} \\
\mathbf{I} \\
\mathbf{R} \\
\mathbf{E} \\
\mathbf{C} \\
\mathbf{T} \\
\mathbf{O}\end{array}$} & $\begin{array}{l}\text { Superficie de área verde por } \\
\text { habitante }\end{array}$ & & & \\
\hline & Calidad del aire & 1 & Muy alto & CORPONARIÑO (S.F), IDEAM (2018) \\
\hline & Calidad del agua superficial & 0,5 & Medio & $\begin{array}{l}\text { CORPONARIÑO (2011) PORH Río } \\
\text { Pasto. }\end{array}$ \\
\hline & Porcentaje de áreas protegidas & 0 & Muy bajo & $\begin{array}{l}\text { Acuerdo Municipal No } 041 \text { del } 29 \text { de } \\
\text { noviembre del } 2010\end{array}$ \\
\hline & $\begin{array}{l}\text { Porcentaje de residuos sólidos } \\
\text { aprovechados }\end{array}$ & 0,8 & Alto & $\begin{array}{l}\text { CORPONARIÑO (2015) Plan de } \\
\text { gestión integral de residuos sólidos } \\
\text { Pasto } 2015 \text { - } 2027 \text {. }\end{array}$ \\
\hline & $\begin{array}{l}\text { Porcentaje de población urbana } \\
\text { expuesta a ruido por encima del } \\
\text { nivel de referencia }\end{array}$ & 0 & Muy bajo & $\begin{array}{l}\text { Este estudio, 2019. Apoyado con } \\
\text { Datos de CORPONARIÑO (2013) }\end{array}$ \\
\hline & $\begin{array}{l}\text { Porcentaje de población que } \\
\text { participa en gestión ambiental } \\
\text { urbana }\end{array}$ & 0,3 & Bajo & Este estudio. \\
\hline & $\begin{array}{l}\text { Porcentaje de población } \\
\text { vinculada a estrategias de } \\
\text { educación ambiental en áreas } \\
\text { urbanas }\end{array}$ & 0,3 & Bajo & Este estudio. \\
\hline & $\begin{array}{l}\text { Porcentaje de población urbana } \\
\text { localizada en zonas de amenaza } \\
\text { alta }\end{array}$ & 1 & Muy alto & $\begin{array}{l}\text { Este estudio, 2019. Apoyado } \\
\text { con Datos de Servicio geológico } \\
\text { colombiano (2015) }\end{array}$ \\
\hline \multirow{5}{*}{$\begin{array}{c}\mathbf{I} \\
\mathbf{N} \\
\mathbf{D} \\
\mathbf{I} \\
\mathbf{R} \\
\mathrm{E} \\
\mathrm{C} \\
\mathbf{T} \\
\mathbf{O}\end{array}$} & $\begin{array}{l}\text { Consumo residencial de agua por } \\
\text { habitante }\end{array}$ & 1 & Muy alto & $\begin{array}{l}\text { Informe Nacional de Calidad } \\
\text { Ambiental Urbana - Ministerio de } \\
\text { Ambiente y Desarrollo Sostenible. } \\
\text { (2016). }\end{array}$ \\
\hline & $\begin{array}{l}\text { Consumo residencial de energía } \\
\text { por habitante }\end{array}$ & 0 & Muy bajo & $\begin{array}{l}\text { Informe de gestión CEDENAR S.A. } \\
\text { E.S.P 2019, CEDENAR (2019), } \\
\text { Ministerio de Ambiente y Desarrollo } \\
\text { Sostenible. (2016). }\end{array}$ \\
\hline & $\begin{array}{l}\text { Porcentaje de residuos sólidos } \\
\text { dispuestos adecuadamente }\end{array}$ & 0 & Muy bajo & $\begin{array}{l}\text { Informe de gestión } 2019 \text { EMAS S.A. } \\
\text { E.S.P, CEDENAR (2019). }\end{array}$ \\
\hline & $\begin{array}{l}\text { Porcentaje de suelos de } \\
\text { protección urbanos }\end{array}$ & 1 & Muy alto & $\begin{array}{l}\text { Este estudio, 2019. Apoyado con } \\
\text { Datos POT - 2014- } 2027 \text { (Alcaldía } \\
\text { de Pasto, 2014) }\end{array}$ \\
\hline & $\begin{array}{l}\text { Espacio público efectivo por } \\
\text { habitante }\end{array}$ & 0 & Muy bajo & $\begin{array}{l}\text { Identificación de áreas óptimas } \\
\text { para cubrir el déficit de espacio } \\
\text { público en las comunas } 3,4 \text { y } 5 \\
\text { dela ciudad de San Juan de Pasto } \\
\text { (Montenegro y Delgado, 2015) }\end{array}$ \\
\hline
\end{tabular}

Fuente: Autores 
La investigación logró calcular 9 indicadores directos y 5 indicadores indirectos, tal como lo sugiere el Ministerio de Ambiente y Desarrollo Sostenible en 2016, asumiendo un nivel moderado de confiabilidad, basado en la consecución de la totalidad de los indicadores ambientales urbanos; sin embargo, se evidenciaron complicaciones para el logro de los indicadores, debido a que alguna información no se clasifica a nivel estratégico de comuna. Asimismo, el Informe Nacional de Calidad Ambiental Urbana (Ministerio de Ambiente y Desarrollo Sostenible, 2016), para el nivel de complejidad de urbes con población entre 100.000 y 500.000 habitantes evidencia que los resultados no reportaron el $100 \%$ de indicadores propuestos. Lo anterior, debido a la ausencia de fuentes de información, información no confiable o que no fue posible validar, así como la falta de acciones para recoger la información que permita medir temas estratégicos de la gestión ambiental de las áreas urbanas.

Liévano y Giraldo (2017), en su estudio de calidad ambiental en la localidad de Kennedy, Bogotá, lograron identificar 13 de los 16 indicadores que propone el Ministerio de Ambiente y Desarrollo Sostenible, 2016 a través de la lista de chequeo. Se evidenció una falencia en la consecución de la información ya que no se logra adquirir información del $100 \%$ de los indicadores propuestos. Esta investigación logra la consecución de la totalidad de los indicadores. Sin embargo, la calidad del aire y consumo residencial de agua y energía por habitante se calculan con información de la ciudad de San Juan de Pasto, esto debido a la complejidad del logro de la información a nivel comunal.

Escobar (2006) infiere que los indicadores de calidad ambiental están correlacionados espacialmente. Sin embargo, los resultados de la calificación de los indicadores ambientales directos e indirectos de la comuna tres son muy variables. De ello se puede señalar que existen problemáticas ambientales en las que se debe hacer énfasis para lograr una calidad ambiental idealizada.

\section{Estimación de la calidad ambiental en la zona de estudio}

La obtención de los indicadores de calidad ambiental da lugar a una estimación cuantitativa del índice de calidad ambiental.

Valor final del grupo de indicadores directos, ecuación 14:

$$
\begin{aligned}
& v F D=(0,5+1+0,5+0+0,8+0,3+0,3+1) * \frac{70}{8}[14] \\
& v F D=38,5
\end{aligned}
$$

Valor final del grupo de indicadores indirectos, ecuación 15:

$$
\begin{aligned}
& v F I=(1+0+0+1+0) * \frac{30}{5}[15] \\
& v F I=12
\end{aligned}
$$

La estimación de la calidad ambiental urbana de la comuna tres de la cuidad de Pasto contempla la incorporación de indicadores directos e indirectos, los cuales están ponderados como se indicó anteriormente. Para estimar el ICAU se realizó la suma de los valores finales en los indicadores directos e indirectos. A continuación, se presenta la estimación del índice de calidad ambiental en la ecuación 16. 


\section{$I C A U=38,5+12[16]$ \\ $I C A U=50,5$}

Con base en la metodología planteada por el Ministerio de Ambiente y Desarrollo Sostenible se estimó como de nivel medio la calidad ambiental de la comuna tres de la ciudad de San Juan de Pasto, ya que se encuentra en rango de 40,1 a 60 puntos. El valor obtenido muestra que la calidad ambiental de la comuna tres es un componente importante territorial urbano, prioritario para entidades gubernamentales y que debe ser acogido por habitantes citadinos con el fin de impulsar estrategias, actividades, normativas y políticas que conlleven a mejorar la calidad ambiental. Confirmando la hipótesis de investigación "Existe la necesidad de formular estrategias que permitan mejorar la calidad ambiental de la comuna tres de San Juan de Pasto, debido a que esta cuenta con indicadores bajos y medios de calidad ambiental".

El Informe Nacional de Calidad Ambiental Urbana del 2016 generado por el Ministerio de Ambiente y Desarrollo Sostenible en 2016 expresa que la ciudad de San Juan de Pasto tiene la mejor calidad ambiental del país en poblaciones entre los 100.000 y 500.000 habitantes, con una calificación de 51,4, ubicándose 0,9 puntos por encima del índice de calidad ambiental de la comuna tres $(50,5)$ determinado en este estudio. Del mismo modo, la comuna tres supera la calificación del ICAU respecto a áreas urbanas con población similar en ciudades como Popayán $(15,5)$, Armenia $(39,7)$, entre otros (Ministerio de Ambiente y Desarrollo Sostenible, 2016). Según Escobar (2006), la distribución espacial de la calidad ambiental por comunas muestra una relación indirecta con la centralidad de la ciudad, siendo las áreas periurbanas las que poseen mejor calidad ambiental, lo anterior se confirma en esta investigación, identificando resultados de calidad media, teniendo en cuenta que la comuna tres de la ciudad de Pasto se encuentra ubicada en zona periférica.

Para elaborar el ICAU, el Ministerio de Ambiente y Desarrollo Sostenible, 2016 consideró sistemas existentes de indicadores ambientales e iniciativas internacionales y locales. Entre los antecedentes más importantes está el Índice de Calidad Ambiental Urbana - ICA, desarrollado para el municipio de Cali por la Universidad del Valle, el cual tiene como ventaja una mayor resolución espacial, puesto que llega al nivel de comuna, localidad o barrio. Tal aspecto da claridad de lo conveniente y adecuado de la implementación del ICAU para la comuna 3 de San Juan de Pasto.

\section{Alternativas que contribuyen a mejorar la calidad ambiental urbana y previenen im- pactos ambientales negativos.}

Con el propósito de mejorar la calidad ambiental del área de estudio, esta investigación propone estrategias en función del área verde por habitante y calidad de vida del habitante, elementos importantes en el estudio, para reducir los impactos de los indicadores con calificaciones bajas y medias, para con ello contribuir al mejoramiento de la calidad de vida de las personas. Según Escobar (2006) en el proceso de formulación de estrategias para mejorar la calidad ambiental, se debe priorizar la formulación de estrategias ambientales que impacten de forma diferencial las distintas zonas de la ciudad. A continuación, en la tabla $N^{\circ} 5$, se muestran las estrategias relacionadas con los indicadores de baja y media calificación. 
Tabla 5. Estrategias para mejorar los indicadores con baja calificación

\begin{tabular}{|c|c|}
\hline Indicadores con baja calificación & Estrategias \\
\hline $\begin{array}{l}\text { Superficie de área verde } \\
\text { por habitante. }\end{array}$ & Sistema de terrazas verdes modulares \\
\hline Calidad del agua superficial & Parque lineal Río Pasto \\
\hline Porcentaje de áreas protegidas & Parque ecológico \\
\hline $\begin{array}{l}\text { Porcentaje de población urbana } \\
\text { expuesta a ruido por encima del } \\
\text { nivel de referencia }\end{array}$ & Movilidad sostenible: Sistema público de Bicicletas. \\
\hline $\begin{array}{l}\text { Porcentaje de población que } \\
\text { participa en gestión ambiental } \\
\text { urbana y porcentaje de población } \\
\text { vinculada a estrategias de } \\
\text { educación ambiental en áreas } \\
\text { urbanas }\end{array}$ & $\begin{array}{l}\text { Adecuación, ornamentación y preservación de áreas } \\
\text { verdes comunitarias. }\end{array}$ \\
\hline $\begin{array}{l}\text { Consumo residencial de energía } \\
\text { por habitante }\end{array}$ & $\begin{array}{l}\text { Campañas de concienciación referentes al gasto } \\
\text { energético y uso de energías renovables }\end{array}$ \\
\hline $\begin{array}{l}\text { Espacio público efectivo } \\
\text { por habitante }\end{array}$ & $\begin{array}{l}\text { Regeneración urbana - Demoliendo autopistas } \\
\text { y construyendo parques. }\end{array}$ \\
\hline
\end{tabular}

Fuente: Autores

Con el fin de formular la estrategia más idónea para el mejoramiento de los indicadores de calidad ambiental de la comuna tres, se realizó una priorización de las alternativas a través de una matriz de priorización con metodología de suma ponderada, tal como se indica a continuación en la Tabla 6. 
Tabla 6. Matriz de priorización.

\begin{tabular}{|c|c|c|c|c|c|c|c|c|c|c|}
\hline \multirow[b]{2}{*}{ Estrategias } & \multicolumn{2}{|c|}{$\begin{array}{c}\text { Impacto } \\
\text { ambiental }\end{array}$} & \multicolumn{2}{|c|}{ Alcance } & \multicolumn{2}{|c|}{ Viabilidad } & \multicolumn{2}{|c|}{$\begin{array}{c}\text { Criterio } \\
\text { financiero }\end{array}$} & \multicolumn{2}{|c|}{ Prioridad } \\
\hline & $\stackrel{0}{\emptyset}$ & $\frac{1}{5}$ & 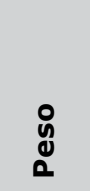 & $\frac{1}{5}$ & : & $\frac{1}{5}$ & @ & $\frac{1}{5}$ & $\stackrel{n}{\stackrel{n}{t}}$ & $\frac{\pi}{\frac{\pi}{0}}$ \\
\hline Terrazas verdes modulares & $44 \%$ & 7 & $21 \%$ & 7 & $19 \%$ & 7 & $17 \%$ & 7 & 7 & 1 \\
\hline $\begin{array}{l}\text { Movilidad sostenible: Sistema público } \\
\text { de bicicletas }\end{array}$ & $44 \%$ & 7 & $21 \%$ & 5 & $19 \%$ & 6 & $17 \%$ & 7 & 6,4 & 2 \\
\hline $\begin{array}{l}\text { Campañas de concienciación referentes } \\
\text { al gasto energético y uso de energías } \\
\text { renovables }\end{array}$ & $44 \%$ & 5 & $21 \%$ & 6 & $19 \%$ & 8 & $17 \%$ & 7 & 6,1 & 3 \\
\hline $\begin{array}{l}\text { Adecuación, ornamentación y } \\
\text { preservación de áreas verdes con la } \\
\text { comunidad }\end{array}$ & $44 \%$ & 5 & $21 \%$ & 7 & $19 \%$ & 7 & $17 \%$ & 5 & 5,8 & 4 \\
\hline Parque lineal Río Pasto & $44 \%$ & 5 & $21 \%$ & 3 & $19 \%$ & 9 & $17 \%$ & 6 & 5,5 & 5 \\
\hline Parque ecológico & $44 \%$ & 6 & $21 \%$ & 5 & $19 \%$ & 5 & $17 \%$ & 4 & 5,2 & 6 \\
\hline $\begin{array}{l}\text { Regeneración urbana /Demoliendo } \\
\text { autopistas y construyendo parques. }\end{array}$ & $44 \%$ & 7 & $21 \%$ & 4 & $19 \%$ & 1 & $17 \%$ & 1 & 4,2 & 7 \\
\hline
\end{tabular}

Fuente: Autores.

Dentro de las estrategias propuestas por esta investigación, se identificaron alternativas que están inmersas dentro del POT Pasto 20142027 (Alcaldía de Pasto, 2014), no obstante, estas no fueron seleccionadas en la priorización de las estrategias, de modo que no se tuvieron en cuenta para formular estrategias que mejoren la calificación de los indicadores de calidad ambiental.

Vidal et al. 2012 indican que la priorización es una acción indispensable, debido a que establece la importancia y aumenta las tasas de éxito. La matriz de priorización por suma ponderada indica que las estrategias que se deben formular son: sistema de terrazas verdes modulares y movilidad sostenible (Sistema público de Bicicletas).

Las estrategias priorizadas en esta investigación son afines al Plan de acción de 2038 de Pasto (Ministerio de Hacienda y Crédito Público. 2015), el cual aportó valiosos insumos para la formulación el POT Pasto 2014 - 2027. Dentro del plan de acción se identifican temas urgentes por desarrollar como la mitigación al cambio climático y el trasporte y movilidad, temas que se abordan desde las estrategias que esta investigación formula. Así como como la sinergia de las mismas estrategias con uno de los retos propuesto por el plan de acción, el cual está encaminado al control del ruido. 


\section{Estrategias de Sistemas de Techos verdes Modulares}

Asegura Osmundson en 2009, que los techos verdes o cubiertas vegetales son cualquier espacio abierto plantado, dispuesto para proveer disfrute humano o una mejora ambiental, que se encuentra separado del suelo por un edificio u otra estructura y que puede estar a la misma altura o por encima del nivel del suelo.

Entre las ventajas que tienen este tipo de techos se incluyen las siguientes: sirven como amortiguadores de sonido, conforman un aislante térmico que aísla la terraza o balcón del calor e impiden que se genere el conocido "efecto de isla térmica" ; capturan y retardan el escurrimiento de aguas de lluvia; traen nuevamente vegetación a zonas urbanas; y prolongan la vida de materiales de techo y paredes (BINAS, 2014). Estos hechos posibilitan la mejora de los indicadores de calidad ambiental, como la superficie de área verde por habitante en San Juan de Pasto, específicamente en la comuna tres.

Esta estrategia está ligada a los temas urgentes por tratar en Municipio, tal como lo identifica el Plan de acción 2038 de Pasto (Ministerio de Hacienda y Crédito Público. 2015), dentro de la sección "Mitigación al Cambio Climático". Asimismo, la estrategia enlaza la protección al medio ambiente y la cultura nariñense, esto en el aspecto de la inclusión de especies nativas, especies ornamentales $y$, especialmente, especies que formen parte de la gastronomía nariñense, especies como el Chamaemelum nobile, Helleborus niger Coriandrum sativum, Solanum betaceum, Allium fistulosum, entre otros.

Para el sistema de techos verdes en la comuna tres, se propone el sistema de bandeja modular, el cual cuenta con una elaboración simple y facilidad de instalación. Cada módulo estándar contiene una jerarquía completa de sistemas de techos verdes que incluyen: drenaje, retención de lluvia, filtración, medios de cultivo y plantas. El módulo está montado directamente en el sistema de impermeabilización del techo, proporcionando protección contra las fluctuaciones UV y térmicas (Guerrero y Gámez, 2014). Estas características viabilizan la estrategia en el área de estudio.

Gracias a su capacidad de retención, los techos verdes pueden causar cambios en la respuesta hidrológica, tales como la reducción del escurrimiento superficial por retención de parte del agua de lluvia (Rosatto, Moyano, Cazorla, Laureda, Meyer, Gamboa, Bargiela, Caso, Villalba, Barrera, Pruzzo, Rodriguez, Oliveri y Waslavky. 2015), hecho que afirman Villalba, Ariel, Rosatto, Bienvenido, Flores-Parra, Botta, Laureda y Perez en 2017, debido a que los resultados de su estudio confirman que el Carpobrutus acinaciformis puede realizar un aporte a la disminución de los escurrimientos urbanos, como vegetación por utilizar en cubiertas naturales para ciudades.

Gómez, Galarza-Molina, y Torres en 2017 mencionan que, la selección de la planta no es únicamente interesante desde el punto de vista paisajístico, sino que puede ser clave desde una perspectiva ingenieril como factor de formulación, puesto que la atenuación de volúmenes y caudales pico en la escorrentía puede contribuir a mitigar amenazas de inundación o racionalizar la infraestructura de drenaje urbano en ciudades de clima tropical andino. Lo anterior afirma la eficiencia del sistema en la reducción del escurrimiento superficial.

A su vez Schiappacasse, Rodríguez, Nektarios, Gaete, y Maturana, en 2017 afirman que los sistemas de techo verde modulares tienen un desarrollo próspero y afianza la eficiencia en la mejora paisajística, por su parte 
Villanueva-Solis, Ranfla, y Quintanilla-Montoya en 2013 indican que los techos verdes ofrecen una eficacia en la reducción de la temperatura que puede llegar a ser hasta de $5,08{ }^{\circ} \mathrm{C}$ en eventos de islas de calor.

\section{Movilidad sostenible - sistema público de bicicletas.}

Según el Plan Maestro Metropolitano de la Bicicleta del Valle de Aburrá (Área Metropolitana del Valle de Aburrá, 2015) la movilidad sostenible implica adoptar un conjunto de políticas, medios físicos, normativos y operacionales que promuevan métodos de transporte con el menor costo ambiental, económico y social, asegurando que, a través de ellos, se logre una mejora en la calidad de vida de los habitantes presentes y futuros del territorio metropolitano. El Área Metropolitana del Valle de Aburrá en 2015 en su plan maestro metropolitano de la bicicleta, menciona que la aplicación de esta estrategia genera múltiples beneficios, entre ellos la reducción de la contaminación ambiental y sonora.

Con el fin de disminuir la presión sonora producida por los sistemas de movilidad en la comuna tres de la ciudad de San Juan de Pasto, se plantea una movilidad que incorpore la bicicleta en los sistemas de movilidad, fomentando su uso por medio de medidas de regulación de tránsito, construcción y/o adecuación de infraestructura, y creación de servicios y equipamientos complementarios que faciliten su uso en condiciones favorables, cómodas y atractivas.

El fomento del uso de la bicicleta es una de las políticas más empleadas para contrarrestar los efectos negativos del uso de automóviles en los grandes centros urbanos. Tema urgente por tratar según en el plan de acción 2038 de Pasto (Ministerio de Hacienda y Crédito Público. 2015) en la sección transporte y movilidad sostenible.
En este contexto, en los últimos 10 años, los sistemas de bicicletas compartidas se han popularizado en múltiples ciudades del mundo (Shaheen y Lipman, 2007). Para Romo y Gómez (2013) las fuentes de ruido pueden dividirse entre aquellas que, por sus altos niveles, pueden dañar el órgano auditivo y otras que, con niveles más bajos, pueden molestar y/o afectar la salud psicosomática del individuo. Esta estrategia busca reducir los niveles de ruido emitido por el parque automotor $y$, sobre todo, mitigar las afectaciones en la salud producidas por el ruido, integrando los beneficios a la salud que causa la incursión a una vida deportivamente activa, aligerando la carga automovilística en la red vial y mejorando la fluidez del tráfico.

Bicicleta club de Catalunya (2009), en su Estudio Sobre el Impacto de la Implantación de Sistemas de Bicicletas Públicas en España, concluye que el análisis de los datos obtenidos demostró una serie de tendencias que se dan en los sistemas de bicicletas públicas españoles. Por ejemplo, se constata que existen muchos tipos de sistemas y que la evolución de los que se perciben como exitosos suele ser hacia la automatización. También se ha notado que no solamente las ciudades medianas y grandes implantan con éxito estos sistemas, sino que también las pequeñas pueden implantar sistemas adaptados a sus medidas que les puedan dar buenos resultados.

\section{CONCLUSIONES}

La comuna tres de San Juan de Pasto tiene una calidad ambiental media. Evidenciando una calidad media en los indicadores de agua superficial, áreas verdes por habitante, porcentaje de población expuesta a ruido por encima del nivel de referencia, población que participa en gestión y educación ambiental, consumo residencial de energía y espacio público efectivo por habitante; y presentando buena calidad 
ambiental, con calificación alta en la calidad de aire, disposición adecuada de residuos sólidos, población urbana localizada en zonas de alta amenaza, consumo residencial de agua y porcentaje de suelos de protección urbanos.

Se formularon y priorizaron estrategias enfocadas a ampliar la superficie de área verde por habitante y reducir el porcentaje de población urbana expuesta a ruido por encima del nivel de referencia; y a mejorar la calidad ambiental y la prevención ante impactos ambientales negativos de la comuna tres de la ciudad de San Juan de Pasto, esto a razón de la multiplicidad de los beneficios brindados por las estrategias.

La metodología del ICAU tiene una viabilidad moderada en la aplicación a nivel estratégico de comuna, ya que reconoce una o varias posibilidades para la identificación de los indicadores de calidad ambiental; sin embargo, se evidenciaron problemáticas en la consecución de información en este estudio y en otras regiones del país. Por lo que se ve condicionado el análisis de la información, debido la heterogeneidad de la misma. Aun cuando la calidad ambiental está determinada por una cantidad amplia de indicadores, el uso de los Sistemas de Información Geográfica favorece y enriquece el estudio del ICAU en diferentes dimensiones espaciales.

\section{CONTRIBUCIÓN DE LA AUTORÍA}

Primer autor: investigación, análisis de datos, escritura - borrador original. Segundo autor: investigación, conceptualización, escritura - revisión y edición. Tercer autor: revisión y edición.

\section{AGRADECIMIENTOS}

A la comunidad académica e investigativa de la Universidad de Nariño, programa de Ingeniería Ambiental, Facultad de Ciencias Agrícolas, que contribuyen en la formación de profesionales idóneos en el campo de la ciencia, tecnología e investigación.

\section{LITERATURA CITADA}

Alcaldía de Pasto (2014). Plan de ordenamiento territorial de Pasto 2014 - 2027: documento técnico soporte. Recuperado de: https:// www.pasto.gov.co/index.php/component/ phocadownload/category/315-planeacion2015?download=6756:cartilla_pot_2014_2027_v1

Alcaldía de Pasto (2014) Plan de ordenamiento territorial de Pasto 2014 - 2027. Cuaderno Diagnóstico Sociocultural. Recuperado de: https:// www.pasto.gov.co/index.php/component/phocadownload/category/397-cuadernos-diagnosticos-pot-2015-2027?download=7984: cuader no_dimension_sociocultural_v4

Alcaldía de Pasto (2015). Actualización plan de gestión integral de residuos sólidos. PGIRS 2015-2027. Recuperado de: https://www.pasto.gov.co/index.php/transparencia/planes-amb ientales?download=14838:actualizacion_plan_ de_gestion_integral_de_residuos_solidos_ pgirs_2015_2027_v3

Área Metropolitana del Valle de Aburrá. (2015). Plan Maestro Metropolitano de la Bicicleta del Valle de Aburrá. Recuperado de https://encicla.metropol. gov.co/Documents/5PMB2030.pdf

Aznar, J \& Guijarro, F. (2012). Nuevos métodos de valoración: modelos multicriterio. 2。 ed, Valencia: Universidad Politécnica.

Banco de ideas de negocios ambientales sostenibles (BINAS). (2014). Diseño y construcción de Green Roofs (Techos verdes). Recuperado de: https:// www.tenerife.es/portalcabtfe/images/PDF/temas/medio_ambiente/TechosVerdesJun15.pdf

Bicicleta Club de Catalunya. (2009). Estudio sobre el impacto de la implantación de sistemas de bicicletas públicas en España. de https:// bicicletapublica.files.wordpress.com/2015/08/ bacc_2009_estudio-sobre-el-impacto-de-la-implantacion-de-sistemas-de-bicicletas-publicasen-espac3b1a.pdf

Bocquier, P. (2005). World Urbanization Prospects: an alternative to the UN model of projection compatible with the mobility transition theory. Demographic Research, 12(9), 197-236. 
Centrales Eléctricas de Nariño S.A. E.S.P. (2019). Informe de Gestión 2019. Recuperado de: https://www.cedenar.com.co/webcedenar/informe-de-gestion/

Charry, M. C., \& Gallego Trujillo, M. A. (2018). Análisis de los obstáculos a los cuatro tipos de innovación tecnológica: caso de las empresas industriales en Colombia (tesis de pregrado). Universidad de La Sabana, Chía, Colombia.

Corporación autónoma regional de Nariño CORPONARIÑO. (2011). Plan de ordenamiento del cauce principal del Rio Pasto. Recuperado de: http:// www.corponarino.gov.co/expedientes/descontaminacion/porhriopasto.pdf

Corporación autónoma regional de Nariño CORPONARIÑO. (2015). Informe de gestión institucional - Vigencia 2014. Recuperado de: https:// docplayer.es/13490011-Informe-de-gestion-institucional.html

Corporación autónoma regional de Nariño CORPONARIÑO. (2016). Plan de Gestión Ambiental Regional del departamento de Nariño 2016 - 2036. Recuperado de: https://corponarino.gov.co/wpcontent/uploads/2016/11/PGAR-2016-2036-VF. $\underline{\mathrm{pdf}}$

Corporación autónoma regional de Nariño CORPONARIÑO. (2013). Sistema vigilancia calidad aire. Recuperado de: http://corponarino.gov.co/ tramites-y-servicios/tramites-ambientales/recurso-aire/sistema-vigilancia-calidad-aire/

Diakoulaki, D., Mavrotas, G., y Papayannakis, L. (1995) Determining objective weights in multiple criteria problems: The critic Method. Computers Operational Research, 22(7), 763-770.

Empresa Metropolitana de Aseo de Pasto S.A. E.S.P (2018) Informe de gestión 2018. Recuperado de: http://www.emaspasto.com.co/ wp-content/uploads/2019/09/INFORME-DEGESTI\%C3\%92N-2018.pdf

Escobar, L. (2006). Indicadores sintéticos de calidad ambiental: un modelo general para grandes zonas urbanas. EURE (Santiago), 32(96), 73-98.

Figueroa, J, \& Ordóñez, W. (2014). Caracterización de la evolución urbana en los barrios de la comuna cinco de la ciudad de San Juan De Pasto periodo 1970-2000 (tesis de pregrado). Universidad de Nariño, San Juan de Pasto, Colombia.

Franco R., J. (2012). Contaminación atmosférica en centros urbanos. Desafío para lograr su sostenibilidad: caso de estudio Bogotá. Revista Escuela De Administración De Negocios, (72), 193-204

Gómez, A., Galarza-Molina, S., y Torres, A. (2018). Propuesta de mejoramiento tecnológico de techos verdes para el clima tropical andino. Ciencia e Ingeniería Neogranadina 28(1), 73-99.

Guerrero, G., \& Gámez, P. (2014). Evaluación y prediseño de cubiertas verdes para la

reutilización de aguas lluvias en la universidad militar nueva granada: una perspectiva desde universidad saludable (Tesis de pregrado). Universidad Militar Nueva Granda, Bogotá, Colombia.

Instituto de Estudios Urbanos - Universidad $\mathrm{Na}$ cional de Colombia. (2017). Debates gobierno urbano. Recuperado de: https://www. institutodeestudiosurbanos.info/observatoriode-gobierno-urbano/publicaciones-de-debatesurbanos/1539-debates-de-gobierno-urbano-18/ file

Instituto de hidrología, meteorología y estudios ambientales IDEAM (2018). Sistemas de vigilancia de calidad del aire. Bogotá, Colombia: Visor geográfico Recuperado de: http://sisaire.ideam. gov.co/ideam-sisaire-web/

Instituto geográfico Agustín Codazzi IGAC (2015). Tan solo el 0,3 por ciento de todo el territorio colombiano corresponde a áreas urbanas. Bogotá: Instituto geográfico Agustín Codazzi. Recuperado de: https://igac.gov.co/es/noticias/tan-soloel-03-por-ciento-de-todo-el-territorio-colombiano-corresponde-areas-urbanas-igac

Liévano, J \& Giraldo, M. (2017) Implementación de la calidad ambiental urbana (ICAU) en la localidad de Kennedy, Bogotá (monografía de especialización). Universidad Piloto de Colombia, Bogotá, Colombia.

Ministerio de Ambiente y Desarrollo Sostenible. (2016). Informe nacional de calidad ambiental urbana - Política de Gestión Ambiental Urbana. Recuperado de: http://observatorio.epacartagena.gov.co/wp-content/ uploads/2016/11/DOCUMENTO_BASE_ICAU_ Actualizacio\%C2\%A6\%C3\%BCn_2016_.pdf

Ministerio de Hacienda y Crédito Público. (2015). Plan de Acción 2038 Pasto, recorrer el buen camino 500 años de tradición. Recuperado de: https://webimages.iadb.org/PDF/ Plan+de+Acci\%C3\%B3n+Pasto+2038.pdf 
Montenegro, J \& Delgado, D. (2015). Identificación de áreas óptimas para cubrir el déficit de espacio público en las comunas 3,4 y 5 de la ciudad de San Juan de Pasto (tesis de pregrado). Universidad de Nariño, San Juan de Pasto, Colombia.

Mundial-DNP. (2014). Política Nacional para Consolidar el Sistema de Ciudades en Colombia: Conpes 3819. Recuperado de: https://colaboracion.dnp. gov.co/CDT/Conpes/Econ\%C3\%B3micos/3819.pdf

Osmundson, T., (1999) Roof Garde History, Desing and Construcción, New York, United States: Norton \& Company Inc.

Pinto, D. (2013). Diseño de un modelo de optimización lineal de logística humanitaria para la ubicación de centros de atención y clasificación de heridos en caso de una erupción volcánica en la ciudad de Pasto (tesis de pregrado). Universidad de los Andes, Bogotá, Colombia.

Rojas, A. (2011). Calidad de vida, calidad ambiental y sustentabilidad como conceptos urbanos complementarios. Revista Venezolana de Sociología y Antropología. 21(61), 183-189.

Romo, J., y Gómez, A. (2013). La percepción social del ruido como contaminante. En M. Aguilar, E. Delgado, V. Vázquez y O. Reyes (Ed.), Ordenamiento territorial y participación social: problemas y posibilidades (pp.271-293). México: Instituto Nacional de Ecología y Cambio Climático.

Rosatto, H., Moyano, G., Cazorla, L., Laureda, D., Meyer, M., Gamboa, P., Bargiela, M., Caso, C., Villalba, G., Barrera, D., Pruzzo, L., Rodriguez Plaza, L., Oliveri, A., y Waslavky, A. (2013). Eficiencia de la retención del agua lluvia de cubiertas vegetadas de tipo "extensivo" e "intensivo". Revista de la Facultad de Ciencias Agrarias de la Universidad Nacional de Cuyo, 45(1), 169-183.
Schiappacasse, F., Rodríguez, E., Nektarios, P., Gaete, K., y Maturana, L. (2017). Growth of the Chilean plants Haplopappus macrocephalus and Selliera radicans on an extensive modular green roof system under three irrigation regimes. Idesia (Arica), 35(3), 31-39.

Servicio Geológico Colombiano (2015). Portal de servicio geológico Colombiano. Mapa de amenazas. https://www2.sgc.gov.co/sgc/volcanes/VolcanGaleras/Paginas/Mapa-de-amenaza.aspx

Shaheen, S. \& Lipman, T. (2007). Reducing greenhouse emissions and fuel consumption: sustainable approaches for surface transportation. IATSS Research, 31(1), $6-20$.

Surveymonkey (S.f.). Tamaño de muestra: calculadora del tamaño de muestra. https:// es.surveymonkey.com/mp/sample-size-calculator/

Vidal, C. (Ed.). (2012). Guía metodológica para la priorización de proyectos: Un enfoque aplicado a la infraestructura, la logística y la conectividad. Cali, Colombia: Editorial Javeriano.

Villalba, G., Ariel, Rosatto, Rosato, H., Bienvenido, F., Flores-Parra, I., Botta, G., Laureda, D., y Perez, D. (2017). Techos verdes: Contribución de Carpobrotus Acinaciformis al manejo integrado de escurrimientos superficiales urbanos. Revista de la Facultad de Ciencias Agrarias de la Universidad Nacional de Cuyo, 49(2), 169-181.

Villanueva-Solis, J., Ranfla, A., y Quintanilla-Montoya, A. (2013). Isla de calor urbana: modelación dinámica y evaluación de medidas de mitigación en ciudades de clima árido extremo. Información tecnológica, 24(1), 15-24.
Conflicto de Intereses Los autores declaran no tener ningún conflicto de intereses 